\title{
High-yield synthesis of silver nanowires for transparent conducting PET films
}

\author{
Gul Naz ${ }^{*}$, Hafsa Asghar ${ }^{1}$, Muhammad Ramzan ${ }^{1}$, Muhammad Arshad ${ }^{*}$, Rashid Ahmed ${ }^{3}$, \\ Muhammad Bilal Tahir ${ }^{4}$, Bakhtiar UI Haq ${ }^{5}$, Nadeem Baig ${ }^{6}$ and Junaid Jalil ${ }^{1}$
}

\section{Full Research Paper}

\section{Address:}

${ }^{1}$ Institute of Physics, Faculty of Science, The Islamia University of Bahawalpur, Baghdad-ul-Jadid Campus, Bahawalpur 63100, Pakistan, ${ }^{2}$ Nanosciences and Nanotechnology Department, National Centre for Physics, Quaid-i-Azam University Islamabad, Pakistan,

${ }^{3}$ Centre for High Energy Physics, The University of Punjab, Lahore, Pakistan, ${ }^{4}$ Department of Physics, Khawaja Fareed University of Engineering and Information Technology, Rahim Yar Khan, Pakistan, ${ }^{5}$ Advanced Functional Materials \& Optoelectronics Laboratory (AFMOL), Department of Physics, Faculty of Science, King Khalid University, Abha 9004, Saudi Arabia and ${ }^{6}$ Center of Research Excellent in Desalination \& Water Treatment, King Fahd University of Petroleum and Minerals, Dhahran 31261, Saudi Arabia

\section{Email:}

Gul Naz* - gul.naz@iub.edu.pk;

Muhammad Arshad* - arshad_pr2002@yahoo.com

* Corresponding author

Keywords:

silver nanowires; high yield; visible luminescence; PET film;

transmittance; sheet resistance

\begin{abstract}
Silver nanowires (AgNWs) with ultrahigh purity and high yield were successfully synthesized by employing a modified facile polyol method using PVP as a capping and stabilizing agent. The reaction was carried out at a moderate temperature of $160{ }^{\circ} \mathrm{C}$ under mild stirring for about $3 \mathrm{~h}$. The prepared AgNWs exhibited parallel alignment on a large scale and were characterized by UV-vis spectroscopy, scanning electron microscopy (SEM), X-ray diffraction (XRD), and PL spectroscopy. The luminescent AgNWs exhibited red emission, which was accredited to deep holes. The SEM results confirmed the formation of AgNWs of 3.3 to $4.7 \mu \mathrm{m}$ in length with an average diameter of about $86 \mathrm{~nm}$, that is, the aspect ratio values of the AgNWs exceeded 45 . An ink consisting of hydroxyethyl cellulose (HEC) and AgNWs was transferred to polyethylene terephthalate (PET) films by simple mechanical pressing. The PET films retained transparency and flexibility after the ink coating. The maximum transmittance value of as-prepared PET films in the visible region was estimated to be about $92.5 \%$ with a sheet resistance value of ca. $20 \Omega$ /sq. This makes the films a potential substitute to commonly used expensive indium tin oxide (ITO) in the field of flexible optoelectronics.
\end{abstract}

Beilstein J. Nanotechnol. 2021, 12, 624-632.

Received: 17 September 2020

Accepted: 16 June 2021

Associate Editor: S. A. Claridge

(C) $2021 \mathrm{Naz}$ et al.; licensee Beilstein-Institut. License and terms: see end of document. 


\section{Introduction}

Several optoelectronic devices, such as solar cells, touch screens, LC displays, organic EL panels, light-emitting diodes, and organic light emitting diodes, use transparent electrodes made of sputtered indium tin oxide (ITO) films [1-3]. These films are widely used because of their high transmittance, low sheet resistance, and high electrical conductivity. Yet, they still have some major drawbacks such as high cost, intrinsic brittleness due to the ceramic nature [4], and toxic composition. Moreover, the sputtering process is time-consuming and sputtering make the films brittle, which limits the application in flexible applications [5]. Numerous materials are under consideration to overcome these challenges. In the past few years, certain materials, such as graphene, carbon nanotubes (CNT), conductive polymers, and metallic nanowires, have been tested commercially as alternative to ITO films for flexible optoelectronic devices [6-9]. Amongst them, graphene and carbon materials, particularly CNTs, display low optical transparency and high sheet resistance owing to their greater tube-tube resistance and lower inherent carrier concentration [10].

Today, noble metal nanomaterials are extensively employed owing to their superior conduction properties [11]. Among them only silver nanowires (AgNWs) films outperform ITO films in term of transmittance and electrical conductivity [12]. AgNWs are important as they offer a possibility to overcome light-matter interaction in the visible region. The optical properties of AgNWs are determined by localized surface plasmon resonance (LSPR), which depends on shape, size, and environment of the material [13]. AgNWs have gained much attention in replacing ITO because of their low-cost solution-based fabrication, flexibility, and high optical transparency [14,15]. A large-scale uniform synthesis of AgNWs with high aspect ratio would be greatly preferred because of better plasmonic and optical properties [16]. An effective synthetic method is still needed for high yields of impurity-free AgNWs.

In order to synthesize silver nanowires several methods have been successfully developed, including ultraviolet irradiation, salt-free solution-based, salt-mediated solution-based, photo reduction, hydrothermal, wet-chemical, template reduction (hard and soft templates), and ultrasonic reduction methods [1722]. Lithographic and hard template methods are used to prepare silver nanowires with well-defined dimensions but both methods produce polycrystalline silver nanowires with rough surface, triggering significant scattering and reducing length propagation, thus affecting the optical properties [23].

The polyol method is considered as the most promising and adaptable method with respect to yield, cost, simplicity, and reaction time for the preparation of AgNWs with higher aspect ratios [24-27]. This method involves the reduction of metallic salts in the presence of a polyol. This synthetic method was first proposed by Figlarz and co-workers for the synthesis of rod-like silver particles. They employed ethylene glycol as polyol to reduce silver salt in the presence of polyvinylpyrrolidone (PVP), which was used a nucleating agent [28]. Silver nanowires with an average diameter of $20 \mathrm{~nm}$ and lengths up to $20 \mu \mathrm{m}$ were synthesized by using a high-pressure polyol method. The transparent film fabricated by these nanowires had a transmittance of $88 \%$ and a sheet resistance of $40 \Omega / \mathrm{sq}$, which is a performance below that of ITO films. Hence, there is a need to modify this polyol method to produce extra-large and highly ordered silver nanowires to outperform ITO films. It is important to note that the polyol synthesis yields impurities in the form of silver nanoparticles [29]. These nanoparticles formed as a by-product of the reaction drastically affect the electrical conductivity and transparency of the silver nanowires network, thus limiting the optoelectronic applications [30,31].

Here, a fast one-pot modified polyol protocol [32] was employed to obtain ultrapure silver nanowires. In this facile synthesis, ethylene glycol was used as a reducing agent in the presence of PVP as capping agent. Silver nitrate and $\mathrm{CuCl}_{2}$ were used as sources of silver and metallic salt, respectively. The resultant nanowires grew 3.3-4.7 $\mu \mathrm{m}$ in length and 75-97 $\mathrm{nm}$ in diameter. A silver nanowire ink was then transferred to PET films whose transmittance was calculated to be up to $92.5 \%$.

\section{Experimental Materials}

All required chemical reagents, that is, silver nitrate $\left(\mathrm{AgNO}_{3}\right)$, ethyl glycol, polyvinylpyrrolidone (PVP), copper chloride $\left(\mathrm{CuCl}_{2}\right)$, hydroxyethyl cellulose (HEC), polyethylene terephthalate (PET) film, acetone, and ethanol, were purchased from Sigma-Aldrich. All of them were of analytical grade and were used as purchased without any purification. Ethylene glycol (EG) was used as a solvent in the reactions.

\section{Preparation of silver nanowires}

For synthesizing silver nanowires (AgNWs) with high aspect ratio and controlled diameter, ethylene glycol (EG) was used as a solvent and also acted as a reducer. Silver nitrate $\left(\mathrm{AgNO}_{3}\right)$ was used as source of silver. The stabilizer used in the reaction was PVP, which also acted as a capping agent. In addition to stabilizing and capping, PVP also prevented the agglomeration of silver nanowires. $\mathrm{CuCl}_{2}$ was used as a salt precursor providing chloride ions. Chloride ions play a vital role in regulating the growth of AgNWs. 
To prepare self-arranged silver nanowires, first, $150 \mathrm{~mL}$ of EG was stirred and heated at $160{ }^{\circ} \mathrm{C}$ for $1 \mathrm{~h}$ in order to remove any residual water from EG. The temperature was controlled by using an oil bath. After $1 \mathrm{~h}$, a trace amount $(0.225 \mathrm{mg})$ of $\mathrm{CuCl}_{2}$ was added into the reaction solution at $160{ }^{\circ} \mathrm{C}$. After 15-20 min, $1.936 \mathrm{~g}$ of PVP dissolved in $5 \mathrm{~mL}$ of EG was added dropwise in the reaction mixture. The addition of PVP lasted for almost half an hour. Eventually, $0.48 \mathrm{~g}$ of $\mathrm{AgNO}_{3}$ dissolved in $5 \mathrm{~mL}$ of $\mathrm{EG}$ was added dropwise in the solution over a period of $1 \mathrm{~h}$. Initially, the addition rate of $\mathrm{AgNO}_{3}$ in the solution was slow, but at the end the injection rate was increased. The stirring rate significantly affected the synthesis of AgNWs. The whole reaction was carried out under mild stirring.

The reaction solution undergoes many color changes after the addition of $\mathrm{AgNO}_{3}$. Just after the addition of a few drops of $\mathrm{AgNO}_{3}$, the solution color changed from transparent to milky gray, then turned to brick red. At the end, the solution color was yellowish white. The yellow color indicated the presence of silver nanoparticles in the solution. The ratio between $\mathrm{AgNO}_{3}$ and PVP used in the reaction greatly affects the synthesis of silver nanowires [32]. In the present reaction, the ratio of PVP to $\mathrm{AgNO}_{3}$ was 4:1. The solution was then cooled to room temperature by the addition of $30 \mathrm{~mL}$ DI water. The cooled mixture was washed through centrifugation twice at $4000 \mathrm{rpm}$, for $10 \mathrm{~min}$ each. After the removal of supernatant, the residual bottom solution showed a silvery shine, which indicated the presence of AgNWs. The final product volume was raised to $0.5 \mathrm{~mL}$ by the addition of DI water. The synthesized AgNWs were then used for various characterizations. The prepared solu- tion was further diluted and used for UV-vis spectroscopy and PL spectroscopy. Another batch of the same reaction solution was dried and crushed into fine powder used for SEM imaging. This modified synthesis method is shown schematically in Figure 1 and produces about $270 \mathrm{mg}$ of AgNWs, which could be considered a high-yield synthesis.

\section{Formulation of AgNW ink and fabrication of transparent conducting films}

A facile method was chosen for synthesizing the AgNW ink, which involved the mixing of an aqueous solution of the adhesive agent HEC with AgNWs [33]. First, a 1.0 wt \% solution of HEC was prepared in $100 \mathrm{~mL}$ of DI water while stirring at room temperature. Then, $15 \mathrm{~mL}$ of HEC solution was mixed with $20 \mathrm{~mL}$ of AgNW solution in ethanol in a separate glass vial under constant stirring. This was followed by mixing another $15 \mathrm{~mL}$ of HEC solution under stirring at room temperature for 2-3 h until the AgNW solution was completely mixed with HEC.

AgNW ink was then transferred to $5 \times 5 \mathrm{~cm}^{2}$ pieces of PET film by mechanical pressing in order to produce a thin and homogeneous layer of the ink on the surface of the PET substrate. Simply, $0.25 \mathrm{~mL}$ of the ink was transferred dropwise on the substrate followed by rolling a stainless steel rod on the PET film for a few minutes. The ink-coated film was then placed in a preheated oven for $3 \mathrm{~min}$ for curing. The curing temperature was $130^{\circ} \mathrm{C}$. A higher curing temperature may disrupt the PET film structure and bend the film. The experimental results revealed that after ink coating, the substrate PET film retained its flexibility and transparency, as shown in Figure 2.

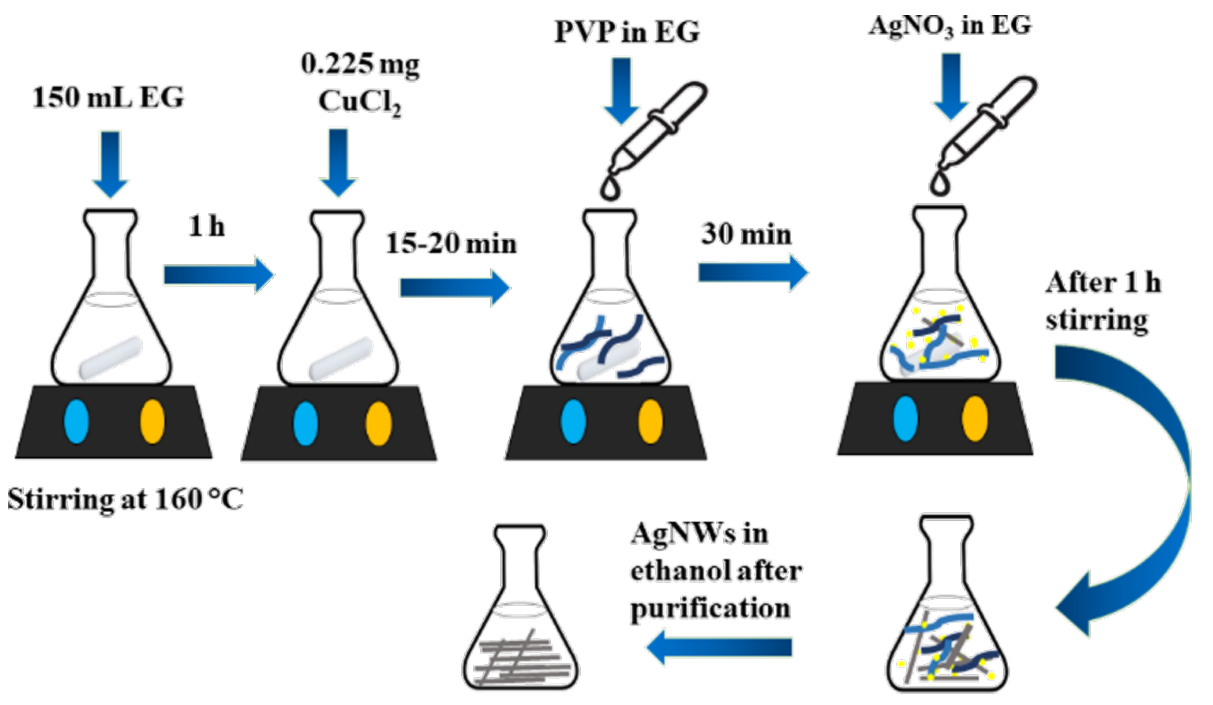

Figure 1: Schematic representation of the modified polyol synthesis of silver nanowires (AgNWs). 

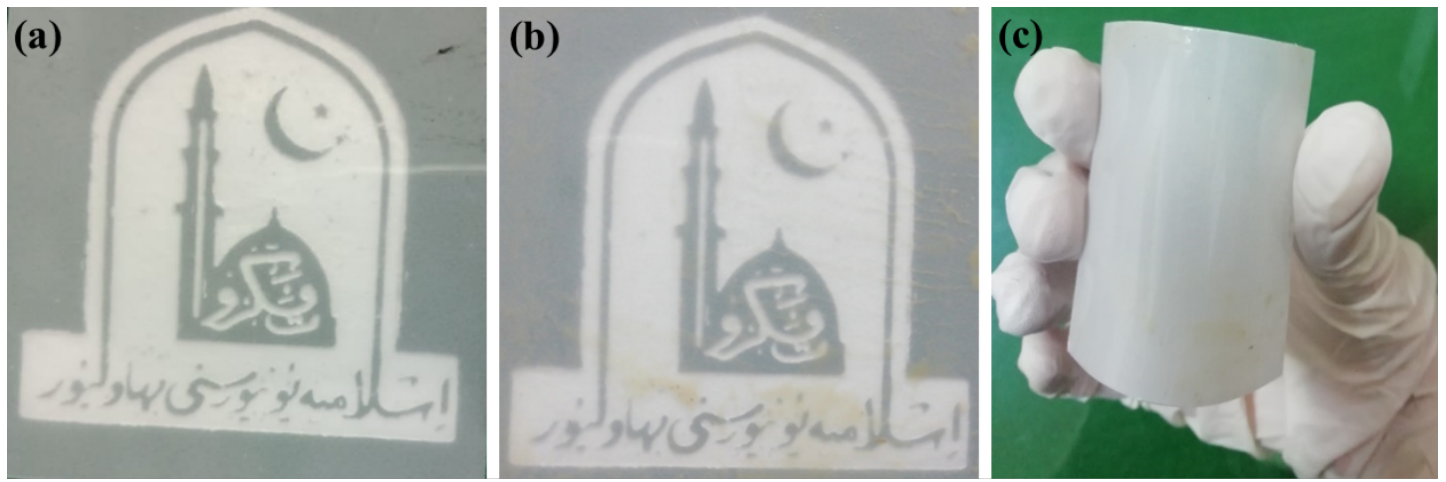

Figure 2: A print of the logo of "The Islamia University of Bahawalpur" behind (a) a PET film without ink coating and (b) a PET film with AgNW ink coating. (c) A photo showing the flexibility of the PET film after AgNW ink coating. These figures clearly depict that PET film retains its flexibility and transparency.

\section{Characterization}

UV-vis absorption spectra of liquid AgNW samples were recorded with a Cecil 7500 double-beam UV-vis spectrophotometer in the 400-800 $\mathrm{nm}$ wavelength range. A Bruker D8 Advance X-ray diffractometer, operating at $35 \mathrm{~mA}$ and $40 \mathrm{keV}$, was used to scan the sample from $20^{\circ}$ to $85^{\circ}$ at room temperature. To examine the surface morphology of as-synthesized powder samples, the scanning electron microscopes (Nova Nano SEM 450 operated at an accelerating voltage of $10 \mathrm{kV}$ and ZEISS SEM operated at an accelerating voltage of $20 \mathrm{kV}$ ) were utilized. Luminescence measurements were carried out by using a Cary Eclipse MY18060003 photoluminescence spectrometer in the wavelength range of 400-700 $\mathrm{nm}$ while exciting the sample at $300 \mathrm{~nm}$ wavelength. The transmissivity values of AgNW-loaded PET films were measured using the UV-vis spectrophotometer in visible light wavelength range. A digital multimeter was used to measure the sheet resistance of the PET films. To test the uniformity and electrical conductivity of the PET films, an electrical circuit including a white LED was utilized.

\section{Results and Discussion}

To determine the morphology, the as-prepared silver nanostructures were first characterized by UV-vis absorption spectroscopy. The absorption spectrum of AgNWs is a function of the dielectric material, the chemicals used, and the particle size [34]. Four samples were examined. First, a sample was taken readily after the addition of $\mathrm{AgNO}_{3}$ to the reaction solution, and the subsequent samples were taken after $10 \mathrm{~min}$ and $20 \mathrm{~min}$, and after washing of the final product (after $60 \mathrm{~min}$ of the reaction). Figure 3 a shows one sharp peak at $401 \mathrm{~nm}$, which confirms the formation of AgNPs in the solution, while the two SPR peaks below $400 \mathrm{~nm}$ are the distinguished feature of AgNWs [35]. As the reaction proceeded to $10 \mathrm{~min}$, the intensi- ty of the absorption peak decreased while a new peak appears at shorter wavelength. This redshift in frequency suggests the growth of rod-like structures, the longitudinal SPR of which might contribute to the advent of a peak at wavelengths shorter than $401 \mathrm{~nm}$. When the reaction approached $20 \mathrm{~min}$, the $\mathrm{Ag}$ nanorods further grew in length, and peak intensities continued to rise and become sharp followed by a gradual redshift of the peak positions. An overall hypsochromatic shift of the two plasmonic bands of AgNWs is accompanied by the enhancement of their aspect ratios. The SPR peak at $365 \mathrm{~nm}$ may be attributed to the plasmon response along the transverse axis of AgNWs, which is identical to that of bulk silver. The second peak at $373 \mathrm{~nm}$ is attributed to the longitudinal plasmon resonance of AgNWs. It is also noted that no other peak was observed, which shows that the final product was free from contamination of any other nanostructures, such as silver nanoparticles or nanocubes. The SEM results also confirm the purity of the product.

The crystalline purity and structure of as-synthesized AgNWs was studied by X-ray diffraction. The different diffraction peaks were matched with the JCPDS card numbers 04-0783 and 99-0094 used for AgNWs. The results clearly indicate that the prepared AgNWs have a face-centered cubic silver crystal structure with the lattice constant $a=4.086 \AA$. The diffraction pattern in Figure $3 \mathrm{~b}$ shows four distinct peaks with $2 \theta$ values of $38.1^{\circ}, 44.30^{\circ}, 64.51^{\circ}$, and $77.43^{\circ}$, corresponding to the (111), (200), (220) and (311) Bragg reflections, respectively. No further peaks were observed. This is a clear indication that the final product is free from impurities and no oxidation of silver has occurred. The crystallite size was calculated using the Scherrer equation. The crystal size was found to be $27 \mathrm{~nm}$, which is smaller than the diameter of the silver nanowires calculated from the SEM results. 

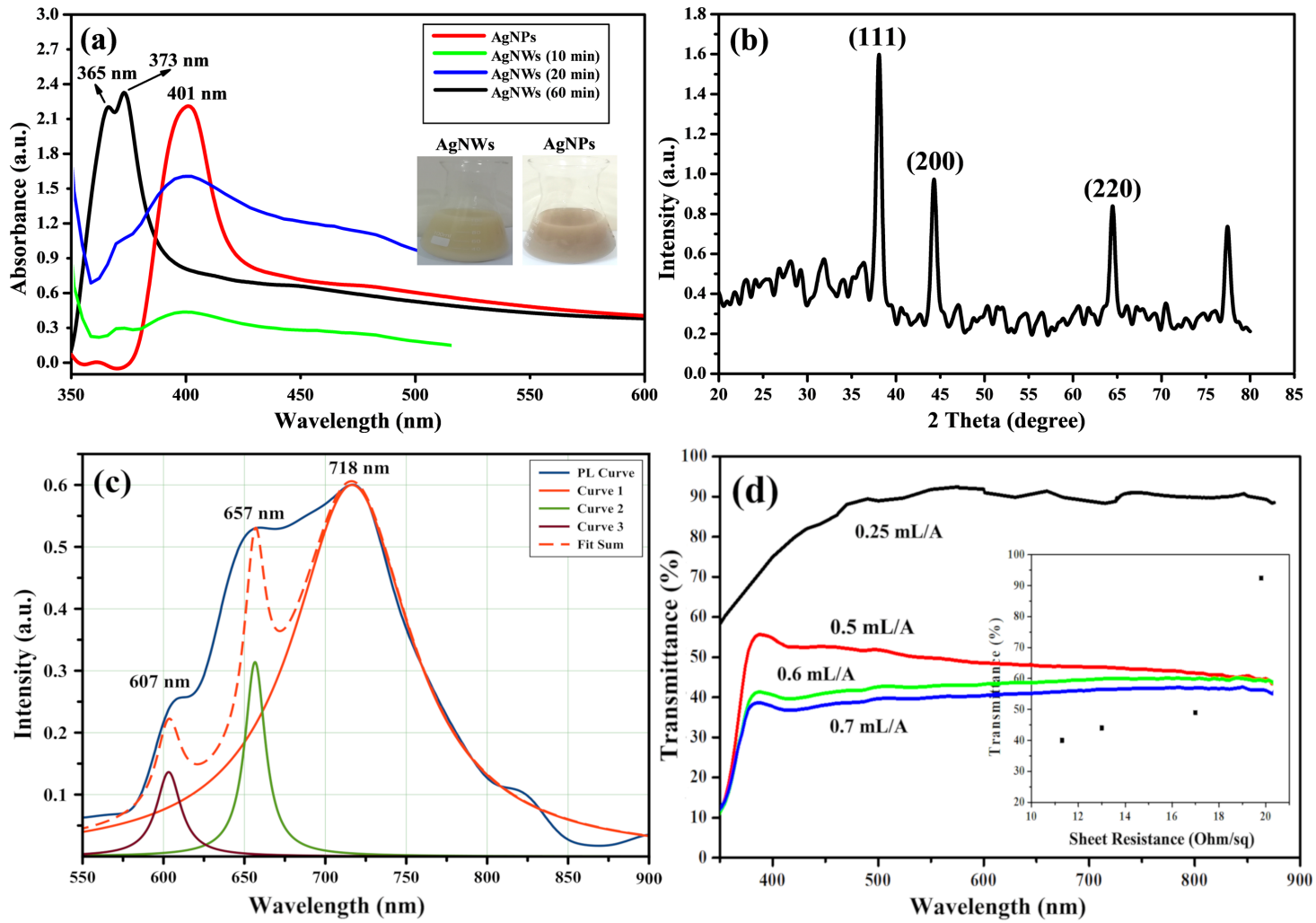

Figure 3: (a) UV-vis absorption spectra indicating the formation of AgNPs just after complete addition of $\mathrm{AgNO}_{3}$ (red line) and the end of the reaction with the formation of AgNWs (black line). The spectra after 10 min (green line) and 20 min (blue line) are also given. The insets showing the actual solution colors. (b) XRD pattern of the as-synthesized AgNWs sample. (c) PL emission spectrum of AgNWs falling in the range of 600-800 nm with red emission. The de-convolution into Lorentzian peaks yields peaks at 607,657 , and $718 \mathrm{~nm}$. The red dashed curve is the fit sum of all three Lorentzian peaks. (d) Transmittance-vs-wavelength plot of AgNW-coated PET films with different densities (corresponding to the quantity of AgNWbased dispersion ink, i.e., $0.25,0.5,0.6$, and $0.7 \mathrm{~mL}$ per unit area $A$ of the film of $5 \times 5 \mathrm{~cm}^{2}$ ) and the inset shows the transmittance-vs-sheet resistance plot of the fabricated PET film samples.

The photoluminescence spectra of silver nanostructures greatly depend on size and shape of the nanostructures. The PL spectrum in the visible region is associated with deep holes. These deep holes cause green, red, and yellow emissions, whereas shallow holes produce blue and violet emissions. AgNPs have been shown to exhibit green emission at about $540 \mathrm{~nm}$ [52]. The PL spectrum of the as-synthesized AgNWs was excited at $300 \mathrm{~nm}$ and the spectrum was observed between 600 and $800 \mathrm{~nm}$. In Figure 3c, the PL spectrum of AgNWs shows a broader PL region with high intensity peaks at 657 and $718 \mathrm{~nm}$. These peaks depict transitions at different energy levels within the bandgap. The AgNWs prepared in this experiment give red emission that attributed to deep holes. Figure $3 \mathrm{~d}$ shows the transmittance spectra of AgNW-loaded PET films. The transmittance $(T)$ of the as-fabricated PET films was determined by using the Beer-Lambert equation:

$$
\% T=\operatorname{antilog}(2-\text { absorbance })
$$

To investigate the effect of the number of coatings on the transparency of the PET film, a range of film samples was prepared with different AgNW ink dispersion volumes (0.25, 0.5, 0.6, and $0.7 \mathrm{~mL}$ ) and characterized as dispersion volume per unit area $(V / A)$, where $A$ represents the unit area of the film of $5 \times 5 \mathrm{~cm}^{2}$. We measured the transmittance $(T)$ at $576 \mathrm{~nm}$ and the sheet resistance $\left(R_{\mathrm{S}}\right)$ for all film samples prepared in this work. The sheet resistance $\left(R_{\mathrm{S}}\right)$ is commonly defined as the resistivity $(\rho)$ of a sheet of material divided by its thickness $(t)$ :

$$
R_{\mathrm{s}}=\frac{\rho}{t}=\frac{\pi}{\ln 2} \cdot \frac{V}{I}
$$

where $V$ is the measured voltage and $I$ is the source current. It was found that for the film with $V / A=0.25 \mathrm{~mL} / A, T$ approached $92.5 \%$ with $R_{\mathrm{S}}$ approaching $19.8 \Omega / \mathrm{sq}$, in contrast to traditional ITO films with lower electrical conductivity and higher transmittance values. For the film with $V / A=0.5 \mathrm{~mL} / A$, $T$ approached $49 \%$ with $R_{\mathrm{S}}=17 \Omega / \mathrm{sq}$. The film with 
$V / A=0.6 \mathrm{~mL} / A$ displayed $T=44 \%$ with $R_{S}=13 \Omega / \mathrm{sq}$, and the film with $V / A=0.7 \mathrm{~mL} / A$ displayed $T=40 \%$ with $R_{\mathrm{S}}=11.3 \Omega / \mathrm{sq}$. These results show that an increase in the number of ink coatings results in a decrease of transmittance and sheet resistance values of the AgNW-coated PET films. A comparison of different parameters of AgNWs synthesized for transparent conducting PET films is presented in Table 1.

The SEM analysis of the as-synthesized AgNWs shows that the nanowires exhibit parallel aligned noodle-like structures over large areas (Figure 4b,c). Along with the reaction temperature of $160{ }^{\circ} \mathrm{C}$, the $\mathrm{AgNO}_{3} / \mathrm{PVP}$ ratio has also great effect on the morphology of AgNWs [53]. In our case, a 1:1 ratio of $\mathrm{AgNO}_{3} / \mathrm{PVP}$ started the growth of $\mathrm{Ag}$ nanoparticles, as can be seen in the SEM image in Figure 4a. Adding more PVP to the reaction promotes the formation of $\mathrm{AgNWs}$. $\mathrm{A} \mathrm{AgNO}_{3} / \mathrm{PVP}$ ratio of 1:4 caused uniform and impurity-free growth of AgNWs. Usually, special attempts are taken to purify the nanowires [39], but in our case no additional steps were required to purify the as-synthesized AgNWs.

The solvent plays a vital role in assembling the AgNWs [54]. It is found that the alignment and density of AgNWs can be easily adjusted by increasing the number of times of water washing. Water, with a very high surface tension of $72.75 \times 10^{-3} \mathrm{~N} \cdot \mathrm{m}^{-1}$ at $20^{\circ} \mathrm{C}$, tends to bundle the hydrophilic AgNWs together [55]. The literature has shown the fabrication of conducting PET films using disordered/unaligned AgNWs. There is a need of fused intersections/connections of nanowires in order to improve the conductivity and transmittance of the PET films [40]. Herein, the end-to-end and side-by-side arranged nanowires with high yield will not require any additional pressing treatments to achieve nanowire interactions. Also, the aligned assembly may not only yield reduced PET film roughness and resistance but also improved transmissivity.

Here, AgNWs with lengths and diameters of 3.3-4.7 $\mu \mathrm{m}$ and 75-97 nm, respectively, have been formed in the reaction. The AgNWs with larger diameters yield lower sheet resistance values if all Ag nanowires in the network are highly connected (at high coverage) [36]. The histogram in Figure 4d shows that some nanowires grow up to $5 \mu \mathrm{m}$ in length. The aspect ratio calculated turns out to be more than 45 . The length of silver nanowires can be controlled predominantly by changing the $\mathrm{PVP} / \mathrm{AgNO}_{3}$ ratio, as reported earlier [32]. It has been suggested that the parallel arrangement of silver nanowires may improve the conductivity and flexibility of PET films coated with AgNW ink. Thus, more studies are required regarding the alignment of AgNWs on the PET film and the impact on the conductivity and flexibility of the PET film. The flexible and

Table 1: A comparison of different parameters of AgNWs synthesized for transparent conducting PET film to present study.

\begin{tabular}{|c|c|c|c|c|}
\hline Synthetic method & Aspect ratio & Transmittance (\%) & Sheet resistance $(\Omega / s q)$ & Ref. \\
\hline one-step synthesis & 2500 & 88.20 & 3.5 & [33] \\
\hline gram-scale Polyol Synthesis & not defined & $<90.0$ & 10.0 & {$[36]$} \\
\hline wet-chemical synthesis & not defined & 85 & 13 & {$[37]$} \\
\hline PVP-mediated polyol process & $30-1000$ & 91.5 & 11.4 & [38] \\
\hline one-pot polyol process & ca. 4000 & 97.70 & 155.0 & [39] \\
\hline laser ablation & not defined & 90 & not defined & [15] \\
\hline solvothermal method & $250-400$ & 89.9 & 58.0 & {$[40]$} \\
\hline polyol synthesis & 2000 & 99.1 & 130.0 & [29] \\
\hline one-pot polyol synthesis & 1009.2 & 81.6 & 11.36 & [28] \\
\hline polyol method & 900 & 96.4 & 24.1 & [41] \\
\hline sonication-induced scission method & ca. 620 & 93.42 & 24.1 & {$[42]$} \\
\hline dynamic heating method using IR light & not defined & 95.0 & 35.0 & [43] \\
\hline polyol process & 625 & 84.0 & 15.2 & {$[44]$} \\
\hline etching liquid-phase synthesis & ca. 500 & 93.0 & 10.0 & {$[45]$} \\
\hline polyol process & not defined & 86.0 & 90.0 & {$[46]$} \\
\hline coating method & ca. 833 & 86.0 & 38.0 & {$[47]$} \\
\hline transfer printing and pressing & ca. 375 & 93.4 & 11.5 & {$[48]$} \\
\hline polyol process & $166-583$ & 81.0 & 130 & [49] \\
\hline bar coating & $444-500$ & 90.3 & 0.745 & {$[50]$} \\
\hline Meyer rod method & $1000-1250$ & $91.8-93.9$ & $14.4-17.6$ & {$[51]$} \\
\hline PVP-mediated polyol method & $>40$ & 92.5 & ca. 20.0 & present \\
\hline
\end{tabular}



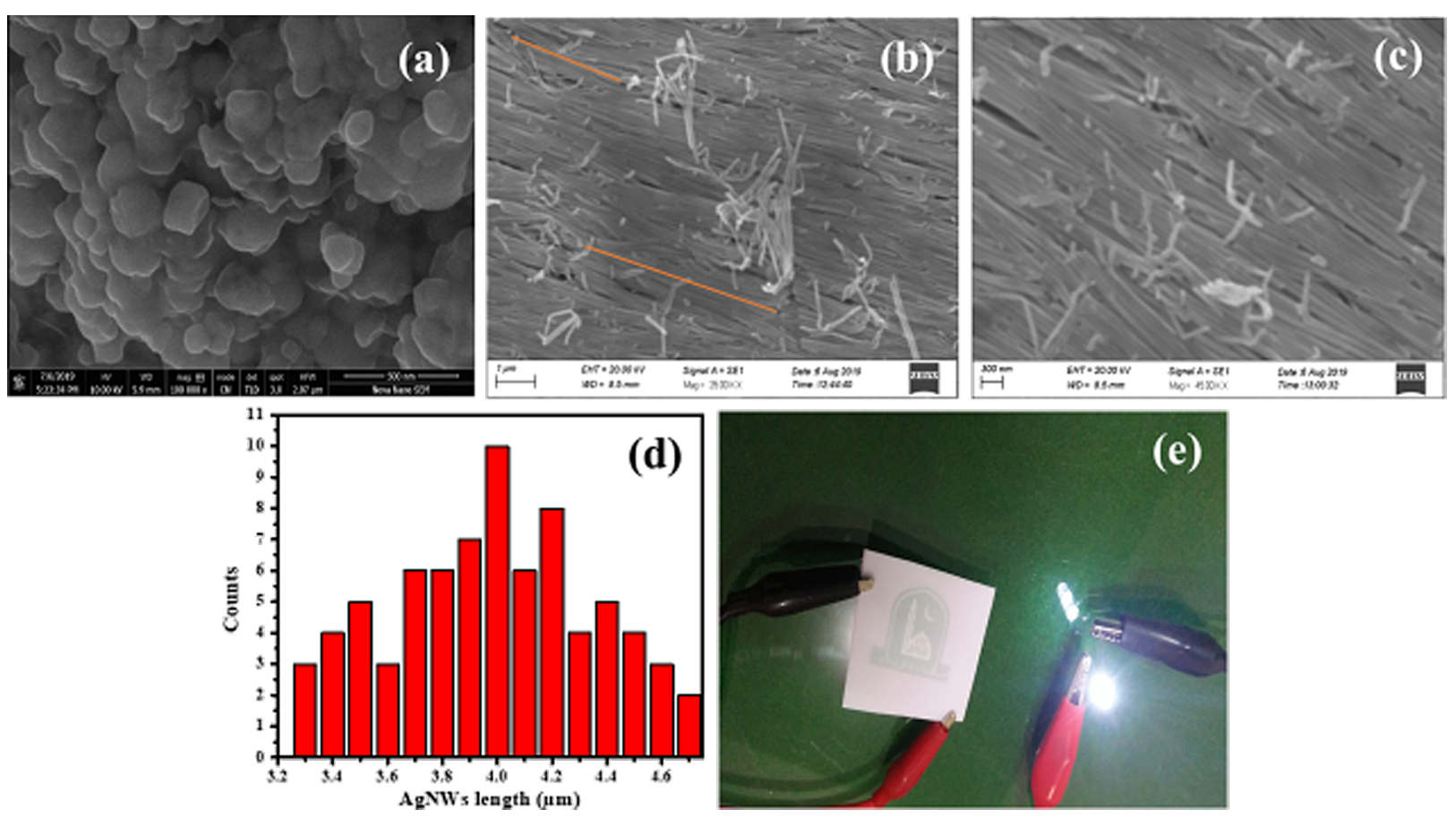

Figure 4: SEM images of (a) AgNPs collected just after complete addition of $\mathrm{AgNO}_{3}$ to the reaction solution and (b, c) $\mathrm{AgNWs}$ in high yield exhibiting parallel alignment over large regions (collected after $60 \mathrm{~min}$ of reaction). (d) Histogram showing the distribution of AgNWs length in the prepared sample. (e) An electric circuit with a lit white LED showing the conductivity of a AgNW-loaded PET film (with sandwiched logo of the institution). The circuit was placed on a glass table with green background.

transparent film was bent to demonstrate its curved surface and flexibility (Figure 2c). To test the electrical conductivity of the film, a white LED was used connected to a pair of batteries and the PET film in series to form a closed circuit (Figure 4e).

To demonstrate the mechanical stability of the AgNW-coated PET films, a fabricated PET film was bent repeatedly by $90^{\circ}$. A test measuring the PET sheet resistance during 500 bend-release cycles was carried out. Figure 5 shows that the PET film has almost maintained its original sheet resistance value after 500 bend-release cycles, thus exhibiting a stable electrical performance. The study describes a simple, inexpensive, eco-friendly and non-toxic method to prepare flexible transparent conducting films that could prove very beneficial for flexible transparent optoelectronic devices and could be used in the place of ITO films.

\section{Conclusion}

A high yield of $100 \%$ pure and aligned arrays of silver nanowires was obtained by using a convenient template-free polyol method. We prepared AgNWs with an average length of $3.3-4.7 \mu \mathrm{m}$ and a mean diameter of approximately $86 \mathrm{~nm}$. The morphology of the Ag NWs was best at a reaction temperature of $160{ }^{\circ} \mathrm{C}$ and a $1: 4$ ratio of $\mathrm{AgNO}_{3} / \mathrm{PVP}$. The XRD analysis confirmed the crystallinity of the AgNWs structures. The

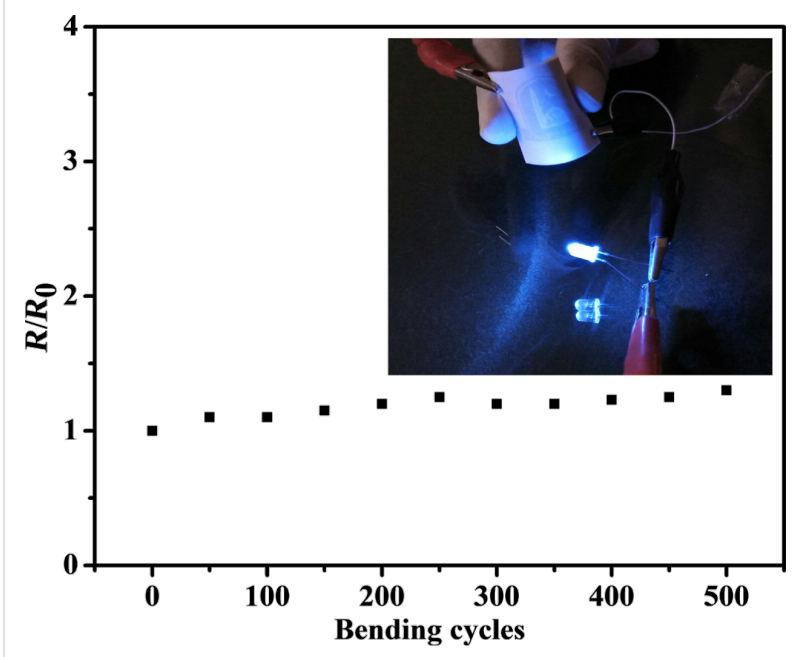

Figure 5: The bending test shows only little change in the sheet resistance of a AgNW-coated PET film after multiple bend-release cycles. The sheet resistance values of the PET film before and after bending test are represented by $R_{0}$ and $R$, respectively. The inset shows that the PET film bent by $90^{\circ}$ is still conducting.

aqueous solution of the AgNWs exhibited a broad PL emission band in the red region. The SEM images confirmed that the final product was free from impurities, that is, silver nanoparticles. No other nanostructures could affect the optical and 
conduction properties, or the roughness of the film. A silver nanowire ink formulated by adding HEC to an aqueous solution of silver nanowires, was then loaded onto the surface of PET films by simple mechanical pressing. The transmittance of the AgNW-coated PET films was calculated to be up to $92.5 \%$ at about $20 \Omega /$ sq sheet resistance. This can make it possible to substitute traditional ITO films in flexible optoelectronics technology.

\section{Funding}

Bakhtiar Ul Haq appreciates the Deanship of Scientific Research at King Khalid University for funding this work through the Research Groups Program under Grant No. R. G. P. 2/126/42.

\section{Acknowledgements}

The author Gul Naz appreciates the support provided by all co-authors from The University of Punjab (Lahore), Khawaja Fareed University of Engineering \& Information Technology (Rahim Yar Khan), National Centre for Physics (Islamabad), King Fahd University of Petroleum \& Minerals (Saudi Arabia) and King Khalid University (Saudi Arabia) in preparation of the manuscript. We are thankful to Materials Chemistry Laboratory (MCL/The IUB) for providing successful PL spectroscopic results.

\section{ORCID ${ }^{\circledR} \mathrm{iDs}$}

Gul Naz - https://orcid.org/0000-0002-8080-8370

Bakhtiar UI Haq - https://orcid.org/0000-0001-9058-2080

Junaid Jalil - https://orcid.org/0000-0003-2658-2240

\section{Preprint}

A non-peer-reviewed version of this article has been previously published as a preprint: https://doi.org/10.3762/bxiv.2020.108.v

\section{References}

1. Margalith, T.; Buchinsky, O.; Cohen, D. A.; Abare, A. C.; Hansen, M.; DenBaars, S. P.; Coldren, L. A. Appl. Phys. Lett. 1999, 74, 3930-3932. doi:10.1063/1.124227

2. Granqvist, C. G.; Hultåker, A. Thin Solid Films 2002, 411, 1-5. doi:10.1016/s0040-6090(02)00163-3

3. Kavei, G.; Gheidari, A. M. J. Mater. Process. Technol. 2008, 208, 514-519. doi:10.1016/j.jmatprotec.2008.01.024

4. Tran, D.-P.; Lu, H.-I.; Lin, C.-K. Coatings 2018, 8, 212 doi:10.3390/coatings8060212

5. Kim, H.; Horwitz, J. S.; Kushto, G. P.; Kafafi, Z. H.; Chrisey, D. B. Appl. Phys. Lett. 2001, 79, 284-286. doi:10.1063/1.1383568

6. Sharma, S.; Shriwastava, S.; Kumar, S.; Bhatt, K.; Tripathi, C. C. Opto-Electron. Rev. 2018, 26, 223-235. doi:10.1016/j.opelre.2018.06.004

7. Liu, Y.; Chen, Y.; Shi, R.; Cao, L.; Wang, Z.; Sun, T.; Lin, J.; Liu, J.; Huang, W. RSC Adv. 2017, 7, 4891-4895. doi:10.1039/c6ra27760h
8. Ma, Y.; Zhi, L. Small Methods 2019, 3, 1800199. doi:10.1002/smtd.201800199

9. Urper, O.; Çakmak, I.; Karatepe, N. Mater. Lett. 2018, 223, 210-214. doi:10.1016/j.matlet.2018.03.184

10. Du, J.; Pei, S.; Ma, L.; Cheng, H.-M. Adv. Mater. (Weinheim, Ger.) 2014, 26, 1958-1991. doi:10.1002/adma.201304135

11. Bi, Y.-G.; Liu, Y.-F.; Zhang, X.-L.; Yin, D.; Wang, W.-Q.; Feng, J.; Sun, H.-B. Adv. Opt. Mater. 2019, 7, 1800778. doi:10.1002/adom.201800778

12. Sim, H.; Bok, S.; Kim, B.; Kim, M.; Lim, G.-H.; Cho, S. M.; Lim, B. Angew. Chem., Int. Ed. 2016, 55, 11814-11818. doi:10.1002/anie.201604980

13. Pourcin, F.; Reynaud, C. A.; Carlberg, M.; Rouzo, J. L.; Duché, D.; Simon, J.-J.; Escoubas, L.; Sauvage, R.-M.; Berginc, G.; Margeat, O.; Ackermann, J. Langmuir 2019, 35, 2179-2187. doi:10.1021/acs.langmuir.8b03003

14. Li, Y.; Guo, S.; Yang, H.; Chao, Y.; Jiang, S.; Wang, C. RSC Adv. 2018, 8, 8057-8063. doi:10.1039/c7ra13683h

15. Cann, M.; Large, M. J.; Henley, S. J.; Milne, D.; Sato, T.; Chan, H.; Jurewicz, I.; Dalton, A. B. Mater. Today Commun. 2016, 7, 42-50. doi:10.1016/j.mtcomm.2016.03.005

16. Madaria, A. R.; Kumar, A.; Zhou, C. Nanotechnology 2011, 22 , 245201. doi:10.1088/0957-4484/22/24/245201

17. Liu, L.; He, C.; Li, J.; Guo, J.; Yang, D.; Wei, J. New J. Chem. 2013, 37, 2179-2185. doi:10.1039/c3nj00135k

18. Bari, B.; Lee, J.; Jang, T.; Won, P.; Ko, S. H.; Alamgir, K.; Arshad, M.; Guo, L. J. J. Mater. Chem. A 2016, 4, 11365-11371. doi:10.1039/c6ta03308c

19. Chen, M.; Wang, C.; Wei, X.; Diao, G. J. Phys. Chem. C 2013, 117, 13593-13601. doi:10.1021/jp404563h

20. Xiang, X.-Z.; Gong, W.-Y.; Kuang, M.-S.; Wang, L. Rare Met. 2016, 35, 289-298. doi:10.1007/s12598-016-0695-6

21. Fahad, S.; Yu, H.; Wang, L.; Zain-ul-Abdin; Haroon, M.; Ullah, R. S.; Nazir, A.; Naveed, K.-u.-R.; Elshaarani, T.; Khan, A. J. Mater. Sci. 2019, 54, 997-1035. doi:10.1007/s10853-018-2994-9

22. Zhang, P.; Wyman, I.; Hu, J.; Lin, S.; Zhong, Z.; Tu, Y.; Huang, Z.; Wei, Y. Mater. Sci. Eng., B 2017, 223, 1-23. doi:10.1016/j.mseb.2017.05.002

23. Ćwik, M.; Buczyńska, D.; Sulowska, K.; Roźniecka, E.; Mackowski, S.; Niedziółka-Jönsson, J. Materials 2019, 12, 721. doi:10.3390/ma12050721

24. da Silva, R. R.; Yang, M.; Choi, S.-I.; Chi, M.; Luo, M.; Zhang, C.; Li, Z.-Y.; Camargo, P. H. C.; Ribeiro, S. J. L.; Xia, Y. ACS Nano 2016, 10, 7892-7900. doi:10.1021/acsnano.6b03806

25. Bi, L.; Yang, Z.; Chen, L.; Wu, Z.; Ye, C. J. Mater. Chem. A 2020, 8, 20030-20036. doi:10.1039/d0ta07044k

26. Jia, C.; Yang, P.; Zhang, A. Mater. Chem. Phys. 2014, 143, 794-800. doi:10.1016/j.matchemphys.2013.10.015

27. Langley, D.; Giusti, G.; Mayousse, C.; Celle, C.; Bellet, D.; Simonato, J.-P. Nanotechnology 2013, 24, 452001. doi:10.1088/0957-4484/24/45/452001

28. Ducamp-Sanguesa, C.; Herrera-Urbina, R.; Figlarz, M. J. Solid State Chem. 1992, 100, 272-280. doi:10.1016/0022-4596(92)90101-z

29. Li, B.; Ye, S.; Stewart, I. E.; Alvarez, S.; Wiley, B. J. Nano Lett. 2015 15, 6722-6726. doi:10.1021/acs.nanolett.5b02582

30. Chen, G.; Bi, L.; Yang, Z.; Chen, L.; Wang, G.; Ye, C. ACS Appl. Mater. Interfaces 2019, 11, 22648-22654. doi:10.1021/acsami.9b04425 
31. Gebeyehu, M. B.; Chala, T. F.; Chang, S.-Y.; Wu, C.-M.; Lee, J.-Y. RSC Adv. 2017, 7, 16139-16148. doi:10.1039/c7ra00238f

32. Yang, H.; Chen, T.; Wang, H.; Bai, S.; Guo, X. Mater. Res. Bull. 2018, 102, 79-85. doi:10.1016/j.materresbull.2018.02.010

33. Li, Y.; Yuan, X.; Yang, H.; Chao, Y.; Guo, S.; Wang, C. Materials 2019, 12, 401. doi:10.3390/ma12030401

34. Zhang, X.-F.; Liu, Z.-G.; Shen, W.; Gurunathan, S. Int. J. Mol. Sci. 2016, 17, 1534. doi:10.3390/jijms17091534

35. Zhou, W.; Hu, A.; Bai, S.; Ma, Y.; Bridges, D. RSC Adv. 2015, 5, 39103-39109. doi:10.1039/c5ra04214c

36. Bergin, S. M.; Chen, Y.-H.; Rathmell, A. R.; Charbonneau, P.; Li, Z.-Y.; Wiley, B. J. Nanoscale 2012, 4, 1996-2004. doi:10.1039/c2nr30126a

37. De, S.; Higgins, T. M.; Lyons, P. E.; Doherty, E. M.; Nirmalraj, P. N.; Blau, W. J.; Boland, J. J.; Coleman, J. N. ACS Nano 2009, 3 , 1767-1774. doi:10.1021/nn900348c

38. Xue, Q.; Yao, W.; Liu, J.; Tian, Q.; Liu, L.; Li, M.; Lu, Q.; Peng, R.; Wu, W. Nanoscale Res. Lett. 2017, 12, 480. doi:10.1186/s11671-017-2259-6

39. Zhang, Y.; Guo, J.; Xu, D.; Sun, Y.; Yan, F. ACS Appl. Mater. Interfaces 2017, 9, 25465-25473. doi:10.1021/acsami.7b07146

40. Liu, B.; Yan, H.; Chen, S.; Guan, Y.; Wu, G.; Jin, R.; Li, L. Nanoscale Res. Lett. 2017, 12, 212. doi:10.1186/s11671-017-1963-6

41. Menamparambath, M. M.; Muhammed Ajmal, C.; Kim, K. H.; Yang, D.; Roh, J.; Park, H. C.; Kwak, C.; Choi, J.-Y.; Baik, S. Sci. Rep. 2015, 5, 16371. doi:10.1038/srep16371

42. Wang, Y.; Yang, X.; Du, D.; Zhao, Y.; Zhang, X. Int. J. Mol. Sci. 2019, 20, 2803. doi:10.3390/ijms20112803

43. Jia, Y.; Chen, C.; Jia, D.; Li, S.; Ji, S.; Ye, C. ACS Appl. Mater. Interfaces 2016, 8, 9865-9871. doi:10.1021/acsami.6b00500

44. Liu, X.; Li, D.; Chen, X.; Lai, W.-Y.; Huang, W. ACS Appl. Mater. Interfaces 2018, 10, 32536-32542. doi:10.1021/acsami.8b10138

45. Tang, L.; Zhang, J.; Dong, L.; Pan, Y.; Yang, C.; Li, M.; Ruan, Y.; Ma, J.; Lu, H. Nanotechnology 2018, 29, 375601. doi:10.1088/1361-6528/aace11

46. Yun, H. D.; Seo, D. M.; Lee, M. Y.; Kwon, S. Y.; Park, L. S. Metals (Basel, Switz.) 2016, 6, 14. doi:10.3390/met6010014

47. Wu, G.; Feng, D.; Yu, J. Integr. Ferroelectr. 2017, 178, 67-72. doi:10.1080/10584587.2017.1324712

48. Jing, M.-x.; Li, M.; Chen, C.-y.; Wang, Z.; Shen, X.-q. J. Mater. Sci. 2015, 50, 6437-6443. doi:10.1007/s10853-015-9198-3

49. Xie, H.; Yang, X.; Du, D.; Zhao, Y.; Wang, Y. Micromachines 2018, 9 , 295. doi:10.3390/mi9060295

50. Shinde, M. A.; Mallikarjuna, K.; Noh, J.; Kim, H. Thin Solid Films 2018, 660, 447-454. doi:10.1016/j.tsf.2018.06.054

51. Wang, Y.; Du, D.; Yang, X.; Zhang, X.; Zhao, Y. Nanomaterials 2019, 9, 904. doi:10.3390/nano9060904

52. Zhang, Z.; Shen, W.; Xue, J.; Liu, Y.; Liu, Y.; Yan, P.; Liu, J.; Tang, J. Nanoscale Res. Lett. 2018, 13, 54. doi:10.1186/s11671-018-2450-4

53. Li, W.; He, Y.; Xu, J.; Wang, W.-y.; Zhu, Z.-t.; Liu, H. J. Alloys Compd. 2019, 803, 332-340. doi:10.1016/j.jallcom.2019.06.257

54. Duan, S.-k.; Niu, Q.--.; Wei, J.-f.; He, J.-b.; Yin, Y.-a.; Zhang, Y. Phys. Chem. Chem. Phys. 2015, 17, 8106-8112. doi:10.1039/c4cp05989a

55. Bi, Y.; Hu, H.; Lu, G. Chem. Commun. 2010, 46, 598-600. doi:10.1039/b911777f

\section{License and Terms}

This is an Open Access article under the terms of the Creative Commons Attribution License (https://creativecommons.org/licenses/by/4.0). Please note that the reuse, redistribution and reproduction in particular requires that the author(s) and source are credited and that individual graphics may be subject to special legal provisions.

The license is subject to the Beilstein Journal of Nanotechnology terms and conditions: (https://www.beilstein-journals.org/bjnano/terms)

The definitive version of this article is the electronic one which can be found at: https://doi.org/10.3762/bjnano.12.51 